\title{
Type-II GaAsSb/GaAsN superlattice solar cells
}

\author{
A. Gonzalo, A. D. Utrilla, U. Aeberhard, J. M. Llorens, B. \\ Alén, et al.
}

A. Gonzalo, A. D. Utrilla, U. Aeberhard, J. M. Llorens, B. Alén, D. Fuertes Marrón, A. Guzman, A. Hierro, J.M. Ulloa, "Type-II GaAsSb/GaAsN superlattice solar cells," Proc. SPIE 10527, Physics, Simulation, and Photonic Engineering of Photovoltaic Devices VII, 105270D (16 February 2018); doi: 10.1117/12.2290079

SPIE. Event: SPIE OPTO, 2018, San Francisco, California, United States 


\title{
Type-II GaAsSb/GaAsN superlattice solar cells
}

\author{
A. Gonzalo ${ }^{\mathrm{a}}$, A. D. Utrilla ${ }^{\mathrm{a}}, \mathrm{U}$. Aeberhard ${ }^{\mathrm{b}}$, J. M. Llorens ${ }^{\mathrm{c}}$, B. Alén ${ }^{\mathrm{c}}$, D. Fuertes Marrón ${ }^{\mathrm{d}}$, \\ A. Guzman ${ }^{\mathrm{a}}$, A. Hierro ${ }^{\mathrm{a}}$, J.M. Ulloa*a \\ ${ }^{a}$ Institute for Systems based on Optoelectronics and Microtechnology (ISOM), Universidad \\ Politécnica de Madrid, Avda. Complutense 30, 28040 Madrid, Spain; ${ }^{b}$ Institut für Energie und \\ Klimaforschung - Photovoltaik (IEK-5), Forschungszentrum Jülich, D-52425 Jülich, Germany; \\ ${ }^{c}$ IMN, Instituto de Micro y Nanotecnología (CNM, CSIC), Isaac Newton 8, PTM, E-28760 Tres \\ Cantos (Madrid), Spain; ' Instituto de Energía Solar (IES), Universidad Politécnica de Madrid, Avda. \\ Complutense 30, 28040 Madrid, Spain
}

\begin{abstract}
Dilute nitride GaAsSbN is an ideal candidate to form the 1-1.15 eV lattice-matched sub-cell that would significantly enhance the performance of 3- and 4-junction solar cells. However, growth problems inherent to this quaternary alloy lead typically to a poor crystal quality that limits its applicability. Better compositional control and crystal quality have been recently reported by growing the material as a $\mathrm{GaAsSb} / \mathrm{GaAsN}$ superlattice, because of the spatial separation of $\mathrm{Sb}$ and $\mathrm{N}$ that avoid miscibility problems. Moreover, these structures provide bandgap tunability trough period thickness. Here we study the performance of lattice-matched $1.15 \mathrm{eV} \mathrm{GaAsSb} / \mathrm{GaAsN}$ type-II superlattice p-i-n junction solar cells with different period thickness and compare them with the bulk and GaAsSbN/GaAs type-I superlattice counterparts. We demonstrate carrier lifetime tunability through the period thickness in the type-II structures. However, the long carrier lifetimes achievable with periods thicker than $12 \mathrm{~nm}$ are incompatible with a high carrier extraction efficiency under short-circuit conditions. Only superlattices with thinner periods and short carrier lifetimes show good solar cell performance. Quantum kinetic calculations based on the non-equilibrium Green's function (NEGF) formalism predict a change in transport regime from direct tunneling extraction to sequential tunneling with sizable thermionic emission components when passing from $6 \mathrm{~nm}$ to $12 \mathrm{~nm}$ period length, which for low carrier lifetime results in a decrease of extraction efficiency by more than $30 \%$.
\end{abstract}

Keywords: type-II superlattice, multi-junction solar cell, carrier lifetime tunability, extraction efficiency

\section{INTRODUCTION}

The dilute nitride alloy GaAsSbN can be grown lattice-matched to GaAs and has a tunable bandgap that covers the 1.0$1.15 \mathrm{eV}$ region. Therefore, it could become a component of monolithically-grown multi junction solar cells, ${ }^{1,2}$ which up to know hold, by far, the record conversion efficiency values: $46.0 \%$ under concentration and $38.8 \%$ under AM1.5G conditions. According to theoretical efficiency calculations, the addition of a sub-cell with an effective bandgap of 1.0$1.15 \mathrm{eV}$ in the standard (Al)InGaP/(In)GaAs/Ge solar cell structure would provide the optimum multi-layer design ${ }^{3,4}$ which would easily surpass the $50 \%$ efficiency if operated under concentration. ${ }^{5}$

One of the attractive characteristics why the GaAsSbN has become a good candidate for such sub-cell is its exceptional band gap versatility, which is explained in the framework of the double band anti-crossing (DBAC) model, ${ }^{6}$ allowing an independent tuning of both conduction and valence band energies by controlling the $\mathrm{N}$ and Sb contents, respectively. ${ }^{7}$ Besides, GaAsSbN remains lattice-matched to GaAs when the condition $[\mathrm{Sb}] \approx 2.8 \times[\mathrm{N}]$ is fulfilled, since $\mathrm{Sb}$ compensates the tensile strain induced by N. Moreover, the two-dimensional growth of the material is facilitated by the surfactant effect of $\mathrm{Sb}$ atoms. ${ }^{8}$ Notwithstanding these facts, important epitaxial growth problems related with the quaternary nature of the GaAsSbN alloy emerge when it is grown as a conventional bulk alloy, such as phase separation (because of its large miscibility gap), clustering, N-related point defects or difficult composition control, that substantially degrade the solar cell performance. ${ }^{9,10,11}$

*jmulloa@isom.upm.es

Physics, Simulation, and Photonic Engineering of Photovoltaic Devices VII, edited by Alexandre Freundlich,

Laurent Lombez, Masakazu Sugiyama, Proc. of SPIE Vol. 10527, 105270D

(C) 2018 SPIE · CCC code: 0277-786X/18/\$18 - doi: 10.1117/12.2290079

Proc. of SPIE Vol. 10527 105270D-1 
An interesting approach to overcome these issues consists on growing the GaAsSbN as a superlattice instead as a conventional alloy. Superlattice structures have already been investigated for solar cells. ${ }^{12,13}$ GaAsSbN-based superlattices can be grown with two different kinds of band alignment, type-I (GaAsSbN/GaAs) or type-II $(\mathrm{GaAsSb} / \mathrm{GaAsN})$, in both cases with electrons confined in GaAsN and holes in GaAsSb. In principle, both kind of superlattices would offer additional advantages as compared to bulk GaAsSbN, such as effective bandgap tunability through period thickness, because of quantum confinement effects. Moreover, type-II GaAsSb/GaAsN superlattices that spatially separates $\mathrm{N}$ and $\mathrm{Sb}$, has the advantage of potentially avoiding the miscibility issues related to the simultaneous coexistence of both $\mathrm{Sb}$ and $\mathrm{N}$ during growth. Indeed, we have recently demonstrated improved compositional control and crystalline quality type-II GaAsSb/GaAsN superlattices as compared to the bulk and type-I GaAsSbN/GaAs superlattice counterparts. ${ }^{14}$ In addition, these type-II structures show long carrier lifetimes that could be beneficial for improved carrier collection.

Nevertheless, the use of superlattices may bring a major drawback, which is carrier trapping and recombination ${ }^{15}$ before the carriers can be extracted. This fact would severely degrade the carrier transport and the solar cell efficiency. In principle, strong electronic coupling and miniband formation would be required to improve carrier transport. Nevertheless, this would also unavoidably affect the radiative carrier lifetime, which again affects significantly solar cell performance. The interplay between radiative lifetime and extraction efficiency would be ultimately determined by the superlattice period thickness, so the design of the superlattice structure has to be considered very carefully.

In this work we compare both experimentally and theoretically the performance of solar cells made of GaAsSbN thick layers, GaAsSbN/GaAs type-I superlattices and $\mathrm{GaAsSb} / \mathrm{GaAsN}$ type-II superlattices. We analyze the structural and optical properties of the different structures and demonstrate radiative carrier lifetime tunability through the period thickness in the type-II samples. The impact of period thickness on carrier extraction efficiency is also analyzed and its influence on solar cell performance is discussed.

\section{EXPERIMENTAL DETAILS}

All the investigated samples were grown by molecular beam epitaxy (MBE) in a Riber 32 machine. The MBE growth process was designed to obtain p-i-n epitaxial structures for subsequent device processing. The substrate is a GaAs (100) $\mathrm{n}+$ wafer; the first layer consisted on a $250 \mathrm{~nm}$ thick GaAs:Si buffer, then the $750 \mathrm{~nm}$ thick active layer made up of the different GaAsSbN-based structures was deposited and finally the structure was completed by a $50 \mathrm{~nm}$ thick GaAs:Be layer on top. The GaAsSbN layer was always grown at a temperature of $470^{\circ} \mathrm{C}$ and with a usual growth rate of $1 \mathrm{ML} / \mathrm{s}$. The nominal $\mathrm{n}$ and p-type doping concentrations were $2 \cdot 10^{18} \mathrm{~cm}^{-3}$ in every case.

The atomic $\mathrm{N}$ flux is provided by a radio frequency plasma source using a $0.1 \mathrm{sccm}$ flow of $\mathrm{N}_{2}$. The $\mathrm{N}$ flux is proportional to the optical emission detection (OED) of the atomic $\mathrm{N}$ into the growth chamber, so the $\mathrm{N}$ flux is easily controlled through the OED. The used $\mathrm{N}$ flux was the same in all samples $(\mathrm{OED}=2.25 \mathrm{~V})$. On the other hand, the $\mathrm{As}_{4}$, $\mathrm{Sb}_{4}$ and $\mathrm{Ga}$ are provided by Kundsen effusion cells. These fluxes were also monitored. All the samples were growth under $\mathrm{As}_{4}$ overpressure conditions with a beam equivalent pressure (BEP) of $1.8 \cdot 10^{-5}$ torr using a $\mathrm{Sb}_{4} \mathrm{BEP}$ of $2 \cdot 10^{-7}$ torr. These $\mathrm{Sb}$ and $\mathrm{N}$ fluxes were chosen after calibrating the composition of ternary ( $\mathrm{GaAsN}$ and $\mathrm{GaAsSb}$ ) and quaternary alloys ${ }^{16}$ so their corresponding nominal contents (approximately $5.0 \% \mathrm{Sb}$ and $1.8 \% \mathrm{~N}$ ) satisfy the lattice matching condition to GaAs $([\mathrm{Sb}] \approx 2.8 \times[\mathrm{N}])$ and give rise to a bandgap energy around $1.15 \mathrm{eV}$.

Four samples with different active layers were grown: a standard quaternary GaAsSbN alloy (sample bulk), a GaAsSbN/GaAs type-I superlattice with $12 \mathrm{~nm}$ period (sample $S L-I_{12}$ ) and two GaAsSb/GaAsN type-II superlattices with $12 \mathrm{~nm}$ and with $6 \mathrm{~nm}$ period (samples $S L-I I_{12}$ and $S L-I I_{6}$, respectively). A sketch of the epitaxial structure of the samples as well as their expected band structure is shown in Figure 1.

The structural properties of the samples were analyzed by High-Resolution X-Ray Diffraction (HR-XRD) with an X'Pert Pro Pan'alytical commercial system using the $\mathrm{Cu}-\mathrm{K} \alpha 1$ line $(1.54056 \AA)$. The optical properties were studied by low temperature $(15 \mathrm{~K})$ photoluminescence $(\mathrm{PL})$ measurements that were carried out using a He-Ne laser. The emitted light was dispersed through a $1 \mathrm{~m}$-spectrometer and detected using a liquid-nitrogen cooled Ge-detector and standard lock-in techniques. Time resolved PL (TR-PL) experiments were performed exciting the sample with $405 \mathrm{~nm}$ pulsed laser light. Decay curves were recorded by a time correlated single photon counting system based on a fast-infrared photomultiplier attached to a $0.3 \mathrm{~m}$-focal length spectrometer. The average excitation power density was $0.6 \mathrm{~W} / \mathrm{cm}^{2}$ at $10 \mathrm{MHz}$. Multiexponential deconvolution analysis was done taking into account the system response measured with a $980 \mathrm{~nm}$ ps laser. 
Time resolution after system response deconvolution is $\sim 200 \mathrm{ps.} \mathrm{Current-voltage} \mathrm{curves} \mathrm{under} \mathrm{AM1.5G} \mathrm{conditions} \mathrm{were}$ measured using an Oriel solar simulator with sample temperature control at $25^{\circ} \mathrm{C}$.

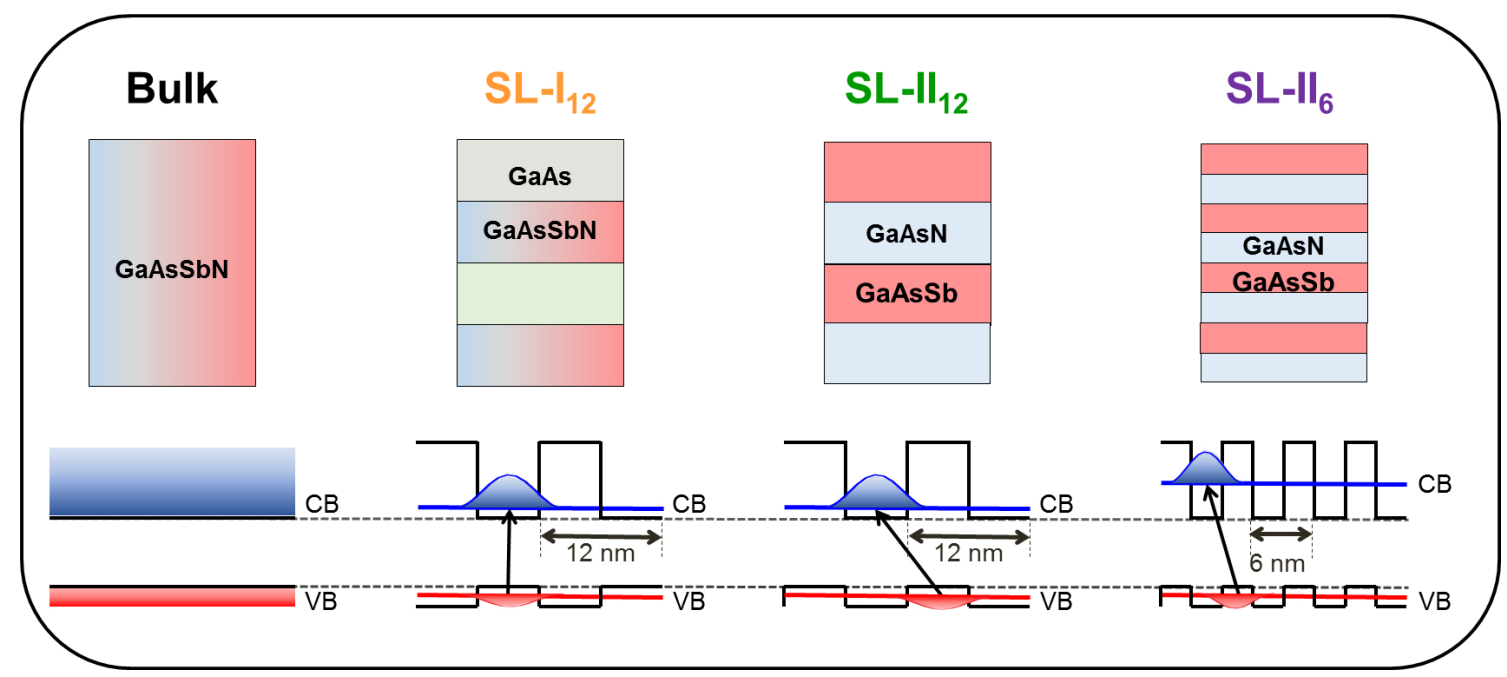

Figure 1. Epitaxial structure and expected band structure of the different samples. A type-I band alignment is expected in sample $S L-I_{12}$, with the maximum of the distribution probability of electron and hole wavefunctions (in blue and red, respectively) localized in the GaAsSbN layer. A type-II band alignment is expected in samples $S L-I I_{12}$ and $S L-I I_{6}$, with maximum of the distribution probability of electron and hole wavefunctions localized in the GaAsN and GaAsSb layers, respectively.

The samples were processed in $200 \mu \mathrm{m}$-diameter mesa-etched devices using standard fabrication techniques. The mesa structures were defined by wet etching using a $\mathrm{H}_{3} \mathrm{PO}_{4}-\mathrm{H}_{2} \mathrm{O}_{2}-\mathrm{H}_{2} \mathrm{O}(1: 1: 8)$ solution. The p-type contact, deposited on top of the mesa, was $\mathrm{Au} / \mathrm{Au}-\mathrm{Zn} / \mathrm{Au}(100 / 800 / 2000 \AA)$. A common n-type contact consisting on Au-Ge/Au (800/2000 $\AA$ ) was evaporated on the substrate side. The contacts were exposed to an annealing process at $400{ }^{\circ} \mathrm{C}$ during 1 minute.

\section{MODEL}

To model photocarrier transport under experimental conditions (finite built-in field and room temperature operation), the NEGF formalism as adapted for photovoltaic device simulation ${ }^{17}$ is used. This approach has the advantage that it captures the essential mechanisms of charge carrier generation, transport and recombination in arbitrary potential profiles and operating conditions, and it has been used successfully to analyze photocarrier transport in $\mathrm{Si}_{-} \mathrm{SiO}_{\mathrm{x}}$ and III-V alloy superlattices. ${ }^{18,19}$ The electronic structure model for the bulk constituent materials used at this stage consists of a simple two band effective mass model with parameters obtained from the double BAC theory. ${ }^{7}$ For the effects of temperature, the inelastic scattering of charge carriers due to coupling to dispersionless polar optical phonons and the elastic interaction with acoustic phonons is considered using the Fröhlich Hamiltonian and the deformation potential formalism, respectively. Photogeneration is described within linear coupling to the classical light field, while for the emission, the incoherent coupling to free field modes is considered. ${ }^{20}$ The band parameters used in the simulation are summarized in Table 1. For the electron-phonon interaction, parameters for bulk GaAs were used, and a Kane energy of $\mathrm{P}_{\mathrm{cv}}{ }^{2} /\left(2 \mathrm{~m}_{0}\right)=$ $28.8 \mathrm{eV}$ was assumed for the light-matter coupling.

Table 1. Band parameters used in the NEGF simulations of GaAsSb/GaAsN superlattices.

\begin{tabular}{|ccccc|}
\hline \multicolumn{5}{c|}{ NEGF BAND PARAMETERS } \\
\hline & $\mathrm{m}_{\mathrm{e}} / \mathrm{m}_{0}$ & $\mathrm{~m}_{\mathrm{h}} / \mathrm{m}_{0}$ & $\mathrm{E}_{\text {gap }}(\mathrm{eV})$ & $\mathrm{E}_{\mathrm{aff}}(\mathrm{eV})$ \\
\hline GaAsN & 0.098 & 0.500 & 1.220 & 4.270 \\
\hline GaAsSb & 0.067 & 0.516 & 1.364 & 4.076 \\
\hline \multicolumn{5}{c}{} \\
\hline
\end{tabular}




\section{RESULTS}

\subsection{Structural analysis by XRD}

All samples were first structurally investigated by HR-XRD. The performed measurements consist on rocking curves (omega-2 theta scans around the (004) GaAs reflection), which are shown in Figure 2. In the bulk and $S L-I 1_{12}$ samples the main diffraction peak appears shifted towards the tensile region of the spectrum, more in bulk than in $S L-I_{12}$. On the other hand, the $S L-I I_{12}$ sample is almost perfectly lattice matched, indicating that the expected contents from the calibration with the ternaries were incorporated. The alternate introduction of $\mathrm{Sb}$ and $\mathrm{N}$ in the growth surface allows for an accurate control of the $\mathrm{Sb}$ and $\mathrm{N}$ composition, in contrast to the altered composition in bulk and GaAsSbN/GaAs structures due to the concomitant presence of $\mathrm{N}$ and $\mathrm{Sb}$. This has been already observed recently in similar samples. ${ }^{14} \mathrm{~A}$ deviation from the lattice-matching condition is also observed in the case of sample $S L-I I_{6}$. A series of thin type-II superlattice samples grown for calibration (not shown) show an accurate composition control within the whole range of periods, so the deviation is likely due to small unwanted variations in the growth conditions. Nevertheless, a subtle effect of period thickness on $\mathrm{Sb}$ incorporation cannot be ruled-out in these structures. ${ }^{21}$

The spacing between subsequent secondary peaks is inversely proportional to the superlattice period thickness. Calculations allow us estimating the period thickness, found to be 11.9 in $S L-I_{12}, 13.4$ in $S L-I I_{12}$ and 6.4 in $S L-I I_{6}$, close to the nominal values. Moreover, the narrower main peak in the superlattices as compared to the broader peak in the bulk structure indicates a better strain and composition homogeneity, which means that composition modulation and clustering effects are significantly reduced in the superlattice structures. Remarkably, the secondary peaks are also narrower and more intense in the type-II superlattices as compared to type-I superlattice, reflecting a higher interface quality when $\mathrm{Sb}$ and $\mathrm{N}$ are incorporated separately.

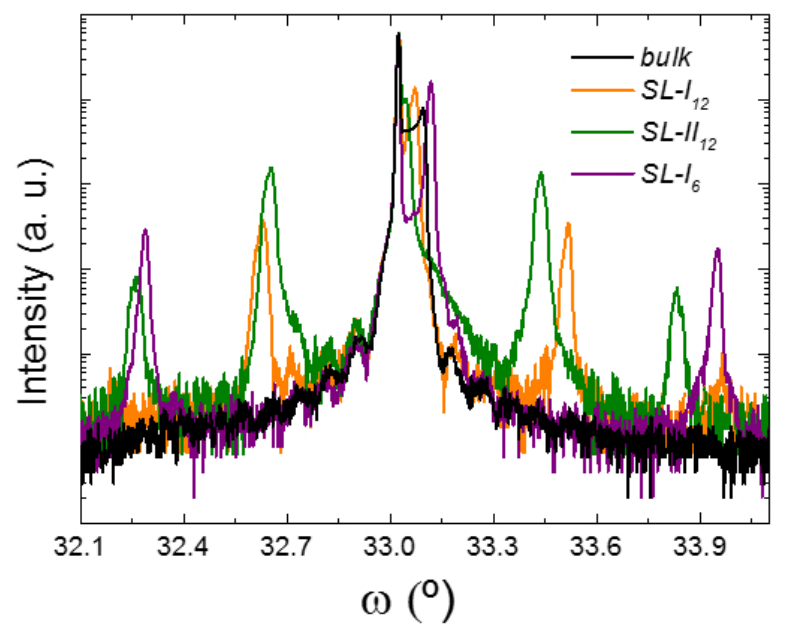

Figure 2. Omega-2 theta scans around the (004) GaAs Bragg reflection performed on the samples bulk, $S L-I_{12}$, SL-II $I_{12}$, and $S L-I I_{6}$. The most intense peak at $33.0239^{\circ}$ corresponds to GaAs.

\subsection{Optical analysis and radiative lifetimes}

Figure 3 shows the $15 \mathrm{~K}$ PL spectra of the four samples. On one hand, the PL peak intensity is larger and the full width at half maximum (FWHM) narrower in the two $12 \mathrm{~nm}$-period superlattices than in the bulk, which could be an indication of improved material quality. On the other hand, comparing $S L-I_{12}$ and $S L-I I_{12}$, the PL peak intensity is higher and the FWHM narrower in the type-II superlattice, which points to a better crystal and interface quality of the $\mathrm{GaAsSb} / \mathrm{GaAsN}$ superlattices. ${ }^{14}$ This results are in agreement with those obtained from XRD. The bulk spectrum appears slightly redshifted, which, taking into account the XRD results, is likely due to a minor increase of $\mathrm{N}$ incorporation. The peak energy of $S L-I I_{6}$ is significantly blueshifted, as expected from the higher quantum confinement due to the reduced period thickness. However, finite element calculations predict a blueshift of $\sim 40 \mathrm{meV}$ when the period is reduced from 13.4 to $6.4 \mathrm{~nm} .{ }^{14}$ This partially explains the measured blueshift of $52 \mathrm{meV}$, the extra shift being likely due to a lack of Sb, which agrees with the position of the main superlattice peak of the XRD spectrum in the tensile region. The reduction of $\mathrm{Sb}$ can be estimated to be $\sim 1 \%$. The period thickness of the type-II superlattices is therefore an additional parameter, besides the 
$\mathrm{Sb}$ and $\mathrm{N}$ contents, to effectively tune material bandgap. The PL blueshift is accompanied by a strong FWHM broadening as well as a reduction of peak intensity, likely due to the formation of broader minibands.

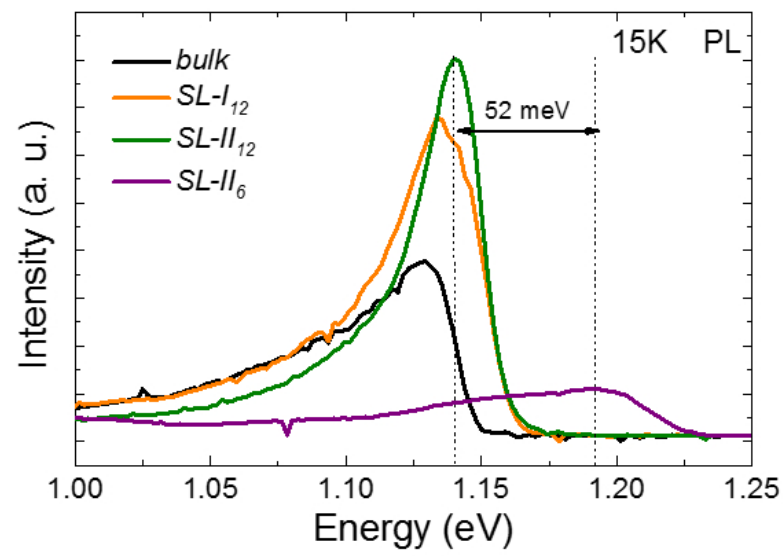

Figure 3. PL spectra taken at low temperature (15 K) of samples bulk, $S L-I_{12}, S L-I I_{12}$, and $S L-I I_{6}$.

TR-PL measurements were carried out in an equivalent series of thinner samples to analyze carrier lifetime and, particularly, in the case of the two type-II superlattices, to obtain information about the influence of period thickness. Figure 4 shows the TR-PL decay curves at the PL peak energy for the whole series of samples. There are clearly two different tendencies in the decay dynamics: on one hand, the $S L-I I_{12}$ has a significantly slower PL decay, while the bulk, $S L-I_{12}$, and even the $S L-I I_{6}$ show a similar faster decay. The bulk sample has the faster PL extinction, followed very closely for the $S L-I_{12}$ and finally for the $S L-I I_{6}$.

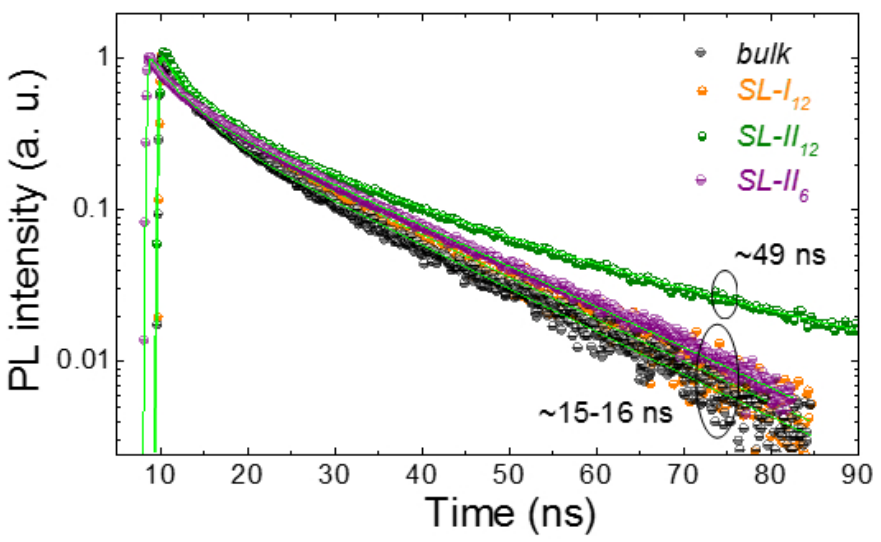

Figure 4. Time-resolved PL decay curves of the samples bulk, $S L-I_{12}, S L-I I_{12}$ and $S L-I I_{6}$ measured at the PL peak energy. Solid lines show the result of a double or triple-exponential fit to the experimental data. The longest times obtained from this fitting for each sample are indicated in the figure.

It was necessary to carry out a multi-exponential fitting analysis in each sample to describe the decay dynamics along the full-time range. The characteristics times and their relative weights obtained from each sample are shown in Table 1. At low temperature the non-radiative recombination is negligible so the measured lifetime is assumed to be the radiative lifetime. Samples bulk, $S L-I_{12}$ and $S L-I_{6}$ show similar decay dynamics, which are accurately described using a double exponential fit that gives two radiative lifetimes of $\sim 3-4 \mathrm{~ns}$ and $\sim 15-16 \mathrm{~ns}$, respectively. However, a longer lifetime with a significant relative weight appears in sample $S L-I I_{12}$. Focusing on the longest radiative lifetimes for each structure, it is three times longer in the $S L-I I_{12}(49 \mathrm{~ns})$ than in the other three cases $\left(16.9,15.5\right.$ and 15.1 in bulk, SL-I $I_{12}$ and $S L-I I_{6}$, respectively). The existence of this significantly longer radiative lifetime only for the type-II superlattice with thicker period confirms the existence of type-II band alignment and slower carrier recombination. On the contrary, when the period thickness is reduced to $6 \mathrm{~nm}$, radiative lifetime is also reduced towards that of conventional type-I structures. This is due to the stronger electronic coupling and formation of minibands when the period thickness is short enough, which 
makes the local density of states less localized (see section 4.4). This demonstrates that the carrier radiative lifetime can be effectively tuned through changing the period thickness in type-II superlattices.

Table 2. PL radiative lifetimes and their relative weights for samples bulk, $S L-I_{12}, S L-I I_{12}$ and $S L-I I_{6}$ obtained from a multiexponential fit to the experimental decay curves measured at the PL peak energy.

\begin{tabular}{|cccc|}
\hline \multicolumn{4}{|c|}{ CARRIER LIFETIMES } \\
\hline & $\tau 1(\mathrm{~ns}) / \mathrm{w} 1(\%)$ & $\tau 2(\mathrm{~ns}) / \mathrm{w} 2(\%)$ & $\tau 3(\mathrm{~ns}) / \mathrm{w} 3(\%)$ \\
\hline$B u l k$ & --- & $15.1 / 76.2$ & $2.9 / 23.8$ \\
\hline$S L-I_{I 2}$ & --- & $15.5 / 78.0$ & $3.3 / 22.0$ \\
\hline$S L-I I_{I 2}$ & $49.1 / 24.6$ & $17.0 / 54.7$ & $4.2 / 20.7$ \\
\hline$S L-I I_{6}$ & --- & $16.7 / 81.5$ & $4.1 / 18.5$ \\
\hline
\end{tabular}

\subsection{Solar cell performance}

The IV curves under AM1.5G conditions of all the samples processed as $200 \mu \mathrm{m}$ diameter mesa-etched devices are shown in Figure 5. The performances of the $S L-I_{12}$ and the $S L-I I_{12}$ are very similar, the type-II showing slightly better efficiency values $\left(1.0 \%\right.$ and $1.1 \%$, respectively.) Both the $S L-I_{12}$ and the $S L-I I_{12}$ have a very low short circuit current density $\left(\mathrm{J}_{\mathrm{sc}}\right)$, slightly above $6 \mathrm{~mA} / \mathrm{cm}^{2}$ and also a very similar open circuit voltage $\left(\mathrm{V}_{\mathrm{oc}}\right), 0.37 \mathrm{~V}$ and $0.38 \mathrm{~V}$, respectively. The $12 \mathrm{~nm}$ period superlattices have weak electronic coupling which results in strong carrier localization, so photogenerated carriers cannot be easily extracted and transport is strongly degraded. These carrier collection problems also likely cause the degradation in the $\mathrm{V}_{\mathrm{oc}}$ as compared to the bulk. The PL spectra shown a bulk effective bandgap smaller than the $12 \mathrm{~nm}$ superlattices ones, thus the $\mathrm{V}_{\text {oc }}$ reduction is still more significant. Therefore, in the case of thick periods, carrier collection problems strongly degrade solar cell performance. On the other hand, switching to reduced period thicknesses results in an impressive enhancement of $\mathrm{J}_{\mathrm{sc}}$, as it is shown by sample $S L-I I_{6}$. This device has a slightly lower $\mathrm{J}_{\mathrm{sc}}$ than the bulk $\left(22.7 \mathrm{~mA} / \mathrm{cm}^{2}\right.$ vs. $25.3 \mathrm{~mA} / \mathrm{cm}^{2}$, respectively) and an efficiency close to that of bulk $(6.2 \%$ and $6.8 \%$, respectively). It is important to notice at this point that the superlattice structure only has half the amount of Sb/Ncontaining material (absorbing material), since only half amount of $\mathrm{N}$ and $\mathrm{Sb}$ atoms are incorporated into the structure. The $S L-I I_{6}$ has higher $\mathrm{V}_{\mathrm{oc}}$ than bulk $(0.45 \mathrm{~V}$ and $0.40 \mathrm{~V}$, respectively), which is slightly lower than the effective bandgap energy increase of $62 \mathrm{meV}$ shown by the PL spectra.

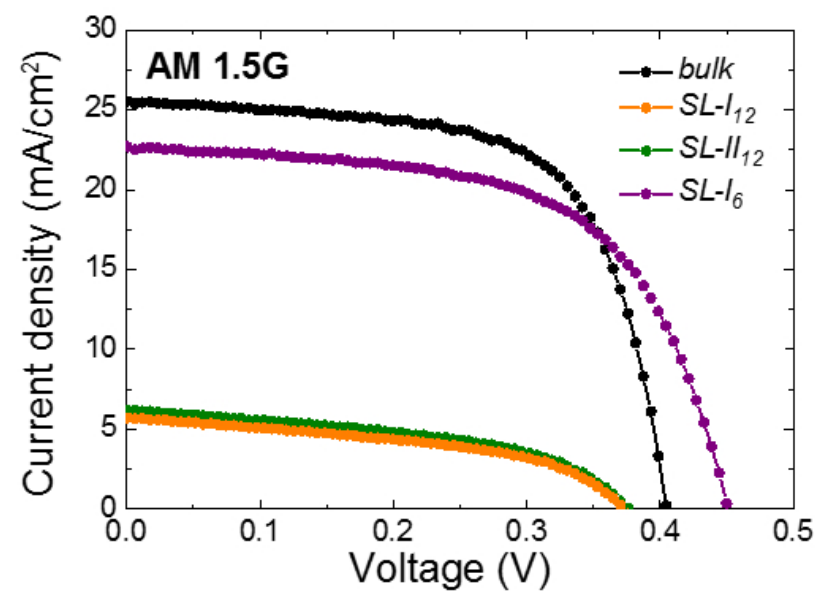

Figure 5. IV curves under AM1.5G conditions for all the series of samples. 


\subsection{NEGF simulation}

Figure 6 shows the NEGF simulation of local density of states and spectral current density at short circuit $\left(\mathrm{V}_{\text {bias }}=0 \mathrm{~V}\right)$ of electrons in GaAsSb/GaAsN type-II SL of period length of $6 \mathrm{~nm}$ and $12 \mathrm{~nm}$, respectively, for monochromatic illumination at $\mathrm{E}_{\gamma}=1.25 \mathrm{eV}$ and $0.1 \mathrm{~kW} / \mathrm{m}^{2}$. The built-in field of $15 \mathrm{kV} / \mathrm{cm}$ corresponds to the situation at short circuit in the $750 \mathrm{~nm}$ active layer samples used to obtain experimental current-voltage characteristics. The 1D LDOS (at zero transverse momentum) shows the transition from wave function delocalization over adjacent wells due to strong coupling at $6 \mathrm{~nm}$ period length to localization within a single QW at $12 \mathrm{~nm}$ period length. As a consequence of the carrier localization, the transport regime reflected in the current spectrum evolves from quasi-ballistic extraction in SL$\mathrm{II}_{6}$ to sequential tunneling between adjacent wells with the emergence of a significant phonon-mediated current component above the top of the barriers in $\mathrm{SL}-\mathrm{II}_{12}$. As the latter processes are significantly slower, the carrier extraction efficiency - computed as the ratio of the terminal current to the integral generation rate - at low carrier lifetime of $\sim 0.01$ ns drops by more than $30 \%$ from SL-II 6 to SL-II 12 .

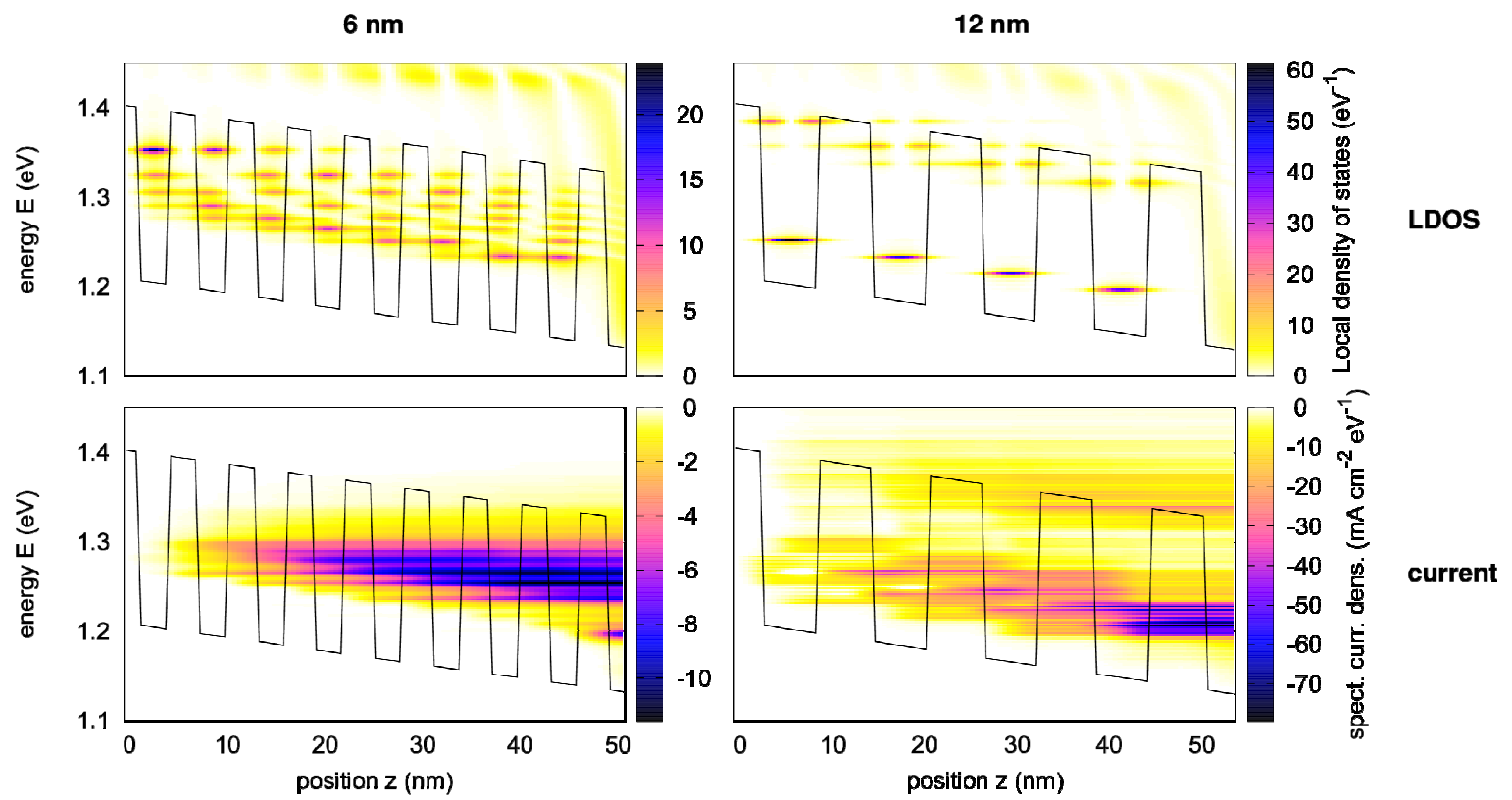

Figure 6. Local density of states (LDOS) and spectral current density in SL-II 6 and SL-II ${ }_{12}$ structures as simulated using NEGF. The 1D LDOS shows the increasing wave function localization with thicker barriers, while the current spectrum reflects the transition in the transport regime from quasi-ballistic extraction in $\mathrm{SL}_{-} \mathrm{II}_{6}$ to sequential tunneling and phononmediated emission over the barrier in $\mathrm{SL}-\mathrm{II}_{12}$.

\section{DISCUSSION}

The GaAsSb/GaAsN superlattice system provides superior material quality as compared to the quaternary bulk and GaAsSbN/GaAs counterparts, which results in a stronger PL emission. These structures show a type-II band alignment and a carrier lifetime which is tunable through the period thickness. Indeed, $\mathrm{GaAsSb} / \mathrm{GaAsN}$ superlattices with periods in the range of $12 \mathrm{~nm}$ show long carrier lifetimes, which in principle could have some advantages for carrier extraction. However, the extraction efficiency in that kind of samples is very low if compared with that of the $\mathrm{GaAsSb} / \mathrm{GaAsN}$ superlattices with thinner periods in the range of $6 \mathrm{~nm}$, as predicted by NEGF simulations. This is due to a transition in the transport regime from quasi-ballistic extraction in $\mathrm{SL}_{-} \mathrm{II}_{6}$ to sequential tunneling and phonon-mediated emission over the barrier in SL- $\mathrm{II}_{12}$. The result is a strong degradation of solar cell performance for thick periods. Nevertheless, $\mathrm{GaAsSb} / \mathrm{GaAsN}$ superlattice solar cells with thin periods below $6 \mathrm{~nm}$ and strong electronic coupling show good 
performance, which makes them good candidates to be series-connected to GaAs/Ge in order to produce more efficient multi-junction solar cells.

\section{ACKNOWLEDGEMENTS}

The authors would like to thank Carlos del Cañizo, from the Solar Energy Institute (IES) from UPM (Spain), for help with the AM1.5G measurements. J. M. Ulloa acknowledges funding from the Spanish MINECO through project MAT2016-77491-C2-1-R and D. Fuertes Marrón from Comunidad de Madrid ( S2013/MAE-2780). U. Aeberhard, D. Fuertes Marrón and J.M. Ulloa acknowledge EU COST Action MP1406 "Multiscale in modelling and validation for solarphotovoltaics (MultiscaleSolar)".

\section{REFERENCES}

[1] Tan, K. H., Loke, W. K., Wicaksono, S., Li, D., Leong, Y. R., Yoon, S. F., Sharma, P., Milakovich, T., Bulsara, M. T. and Fitzgerald, E. A., "Study of a $1 \mathrm{eV}$ GaNAsSb photovoltaic cell grown on a silicon substrate," Appl. Phys. Lett. 104(10), 103906 (2014).

[2] Maros, A., Faleev, N., King, R. R. and Honsberg, C. B., "Growth and characterization of GaAs1$\mathrm{x}-\mathrm{ySbxNy} / \mathrm{GaAs}$ heterostructures for multijunction solar cell applications," J. Vac. Sci. Technol. B 3(2), 02L106 (2016).

[3] Kurtz, S. R., Myers, D. and Olson, J. M., "Projected performance of three and four junction devices using GaAs and GaInP," Conf. Rec. IEE Photovolt. Spec. Conf. 26, 875-878 (1997).

[4] Toprasertpong, K., Fujii, H., Thomas, T., Führer, M., Alonso-Álvarez, D., Farrell, D. J., Watanabe, K., Okada, Y., Ekins-Daukes, N. J., Sugiyama, M. and Nakano, Y., "Absorption threshold extended to $1.15 \mathrm{eV}$ using InGaAs/GaAsP quantum wells for over-50\%-efficient lattice-matched quad-junction solar cells," Prog. Photovoltaics Res. Appl. 24(4), 533-542 (2016).

[5] Tanabe, K., "A review of ultrahigh efficiency III-V semiconductor compound solar cells: multijunction tandem, lower dimensional, photonic up/down conversion and plasmonic nanometallic structures," Energies 2(3), 504530 (2009).

[6] Shan, W., Walukiewicz, W., Ager III, J. W., Haller, E. E., Geisz, J. F., Friedman, D. J., Olson, J. M. and Kurtz, S. R., "Band anticrossing in GaInNAs alloys," Phys. Rev. Lett. 82(6), 1221-1224 (1999).

[7] Lin, Y. T., Ma, T. C., Chen, T. Y. And Lin, H. H., "Energy gap reduction in dilute nitride GaAsSbN," Appl. Phys. Lett. 93(17), 171914 (2008).

[8] Cheah, W. K., Fan, W. J., Yoon, S. F., Tan, K. H., Liu, R. and Wee, A. T. S., "Surfactant and impurity properties of antimony on GaAs and GaAs1-xNx on GaAs (100) by solid source molecular beam epitaxy" Thin Solid Films 488(1), 56-61 (2005).

[9] Harmand, J. C., Caliman, A., Rao, E. V. K., Largeau, L., Ramos, J., Teissier, R., Travers, L., Ungaro, G., Theys, B. and Dias, I. F. L., "GaNAsSb: how does it compare with other dilute III-V-nitride alloys?," Semicond. Sci. Technol. 17(8), 778-784 (2002).

[10] Buyanova, I. A., Chen, W. M. and Tu, C. W., "Magneto-optical and light-emission properties of III-As-N semiconductors," Semicond. Sci. Technol. 17(8), 815-822 (2002).

[11] Chauveau, J. M., Trampert, A., Pinault, M. A., Tournié, E., Du, K. and Ploog, K. H., "Correlations between structural and optical properties of GaInNAs quantum wells grown by MBE," J. Cristal Growth 251(1), 383387 (2003).

[12] Wu, P. H., Su, Y. K., Chen, I. L., Chiou, C. H., Hsu, J. T. and Chen, W. R., "Strain-compensated GaAsN/InGaAs superlattice structure solar cells,"Jpn. J. Appl. Phys. 45(25), 647-649 (2006).

[13] Bhusal, L. and Freundlich, A., "GaAsN/InAsN superlattice based multijunction thermophotovoltaic devices," J. Appl. Phys. 102(7), 074907 (2007).

[14] Gonzalo, A., Utrilla, A. D., Reyes, D. F., Braza, V., Llorens, J. M., Fuertes Marrón, D., Alén, B., Ben, T., González, D., Guzman, A., Hierro, A. and Ulloa, J. M., "Strain-balanced type-II superlattices for efficient multijunction solar cells," Sci. Rep. 7, 4012 (2017).

[15] Toprasertpong, K., Kasamatsu, N., Fujii, H., Kada, T., Asahi, S., Wang, Y., Watanabe, K., Sugiyama, M., Kita, T. and Nakano, Y., "Microscopic observation of carrier-transport dynamics in quantum-structure solar cells using a time-of-flight technique," Appl. Phys. Lett. 107, 043901 (2015). 
[16] Braza, V., Reyes, D. F., Gonzalo, A., Utrilla, A. D., Ben, T., Ulloa, J. M. and González, D., "Sb and N incorporation interplay in $\mathrm{GaAsSbN} / \mathrm{GaAs}$ epilayers near lattice-matching condition for $1.0-1.16-\mathrm{eV}$ photonic applications," Nanoscale Res. Lett. 12, 356 (2017).

[17] Aeberhard, U., "Theory and simulation of quantum photovoltaic devices based on the non-equilibrium Green's function formalism," J. Comput. Electron. 10(4), 394-413 (2011).

[18] Aeberhard, U., "Theory and simulation of photogeneration and transport in Si-SiOx superlattice absorbers," Nanoscale Res. Lett. 6, 242 (2011).

[19] Aeberhard, U., "Simulation of nanostructure-based and ultra-thin film solar cell devices beyond the classical picture," J. Photonics Energy 4(1), 042099 (2014).

[20] Aeberhard, U., "Quantum-kinetic theory of steady-state photocurrent generation in thin films: Coherent versus incoherent coupling," Phys. Rev. B 89, 115303 (2014).

[21] Reyes, D. F., Braza, V., Gonzalo, A., Utrilla, A., Ulloa, J. M., Ben, T. and González, D., "Modelling of the Sb and N distribution in type II GaAsSb/GaAsN superlattices for solar cell applications," Appl. Surf. Sci. (2017). 